\title{
La grande mobilité géographique domicile-travail : l'inscription spatiale des inégalités entre travailleurs
}

Long-Distance Home-Work Employee Mobility : A Spatial Inscription of Inequalities

\section{Thomas Sigaud}

\section{(2) OpenEdition Journals}

Édition électronique

URL : http://journals.openedition.org/travailemploi/9617

DOI : 10.4000/travailemploi.9617

ISSN : 1775-416X

Éditeur

DARES - Ministère du Travail

Édition imprimée

Date de publication : 2 décembre 2019

Pagination : 75-102

ISSN : 0224-4365

Référence électronique

Thomas Sigaud, «La grande mobilité géographique domicile-travail : I'inscription spatiale des

inégalités entre travailleurs », Travail et Emploi [En ligne], 160 | 2019, mis en ligne le 01 novembre 2020, consulté le 25 mars 2021. URL : http://journals.openedition.org/travailemploi/9617 ; DOI : https:// doi.org/10.4000/travailemploi.9617 


\title{
La grande mobilité géographique domicile-travail : l'inscription spatiale des inégalités entre travailleurs
}

\author{
Thomas Sigaud
}

\begin{abstract}
Alors que se multiplient les injonctions à la mobilité géographique des travailleurs, les grandes mobilités domicile-travail sont encore mal connues et souvent ramenées à un simple arbitrage professionnel. En exploitant le Recensement de la population 2015, l' article propose une définition de la grande mobilité domicile-travail qui permet d'identifier, décrire et localiser près de 500000 grands mobiles en France métropolitaine. Loin d'être une ressource univoque qui serait au service des travailleurs, la grande mobilité reproduit les segmentations socioprofessionnelles et spatiales qui structurent les inégalités entre travailleurs et révèle le jeu notamment des inégalités de genre.
\end{abstract}

\begin{abstract}
A vec la crise économique de 2009, le chômage de masse persistant et la fragilisation de l'emploi à l'œuvre depuis le début des années 1980 se sont doublés d'un phénomène d'accentuation des inégalités économiques entre territoires. Le diagnostic largement repris d'une « fracture territoriale » (DAVEZIES, 2012) opposant les métropoles dynamiques aux territoires périphériques en déclin s'est traduit en une recommandation désormais bien intégrée aux politiques de l'emploi : l'économie française aurait besoin d'une plus grande mobilité géographique des travailleurs. S'appuyant sur des théories économiques comme celles du job search, selon laquelle la mobilité géographique serait une ressource dont la valorisation sur le marché du travail compenserait les coûts, une abondante littérature grise prône l'idée selon laquelle une plus grande mobilité géographique des actifs permettrait d'améliorer le fonctionnement du marché du travail et de réduire structurellement le chômage. Ces prescriptions s'appuient notamment sur l'idée largement diffusée selon laquelle il existerait en France des centaines de milliers d' « emplois vacants » attendant d'être pourvus faute notamment d'un appariement géographique correct entre l'offre et la demande de travail (CONSEIL D'ORIENTATION POUR L'EMPLOI, 2013). La mise en avant du
\end{abstract}

\footnotetext{
* Université de Tours, CITERES (UMR 7324, équipe Cost), chercheur associé au Cnam, Centre d'études de l'emploi et du travail (CEET) ; thomas.sigaud@univ-tours.fr.
} 
manque supposé de mobilité géographique des travailleurs en France n'est pas nouvelle (MoscovicI, 1959) mais elle s'inscrit aujourd'hui dans l'évolution des politiques de l'emploi qui visent désormais à mettre en mouvement les individus (Fol et al., 2014) pour répondre aux exigences croissantes de flexibilité et d'adaptabilité propres aux formes contemporaines de mise au travail (BOLTANSKI, CHIAPELLO, 1999). En ramenant la situation des actifs les plus vulnérables à leur manque de mobilité, ce discours et ces politiques participent d'un «paradigme de l'incitation » (GOMEL, MÉDA, 2014, p. 2) dont l'objectif est de modifier les comportements individuels en subordonnant les modes de vie aux politiques de l'emploi. La mobilité géographique est alors présentée comme une ressource univoque à même de sécuriser les trajectoires professionnelles ; elle est ramenée à un arbitrage simplifié à l'extrême entre mobilité et emploi d'une part, et immobilité et chômage d'autre part ${ }^{1}$. Le rapport d'évaluation sur la mobilité géographique des travailleurs établi par l'Inspection générale des finances (IGF) et l'Inspection générale des affaires sociales (Igas) en janvier 2016 (AMARA et al., 2016) présente ainsi la mobilité géographique comme un outil au service de l'amélioration du fonctionnement du marché du travail et comme un dispositif dont il s'agit d'encourager l'usage en la sécurisant et en levant les « freins » et les « obstacles » qui l'empêchent.

Inscrites dans une logique instrumentale et rationnelle qui fait de la mobilité un simple moyen d'étendre et de rendre plus efficace la recherche d'emploi, ces injonctions disqualifient d'emblée toute forme d'immobilité et invisibilisent les arbitrages privés, résidentiels et familiaux mis en jeu par la mobilité géographique. Pourtant, la grande mobilité domicile-travail ne peut pas se ramener à sa seule dimension professionnelle : elle recouvre des arbitrages déterminés par la vie familiale et les choix résidentiels des individus. Paradoxalement peut-être, les individus les plus mobiles sont les plus ancrés : s'ils acceptent et font accepter à leurs proches une grande mobilité domicile-travail, c'est aussi pour ne pas avoir à déménager et ainsi protéger leur ancrage résidentiel (KAUFMANN, 2017). D'une part la grande mobilité domicile-travail n'est pas nécessairement une stratégie rationnelle mise en œuvre par les individus dans le cadre d'un arbitrage entre localisation du lieu de résidence et localisation du lieu de travail (AxISA et al., 2012), et d'autre part ce ne sont pas toujours les contraintes d'accès à l'emploi qui l'emportent dans cet arbitrage : les individus concernés peuvent ne pas considérer le déménagement comme une option (ÖHMAN, LiNDGREN, 2003) ou bien chercher un meilleur équilibre entre vie professionnelle et familiale en s'assurant que leur trajectoire résidentielle ne soit pas déterminée par leurs choix professionnels (KLIS, KARSTEN, 2009).

La grande mobilité domicile-travail doit donc être étudiée comme le produit de choix professionnels profondément imbriqués dans les choix résidentiels et dans la vie personnelle et familiale des individus. Les recherches empiriques portant sur la

1. Cette conception du lien entre mobilité individuelle et emploi a été brièvement mise sur le devant de la scène médiatique lorsque le 4 octobre 2017, Emmanuel Macron avait invité de manière assez crue les salariés de l'équipementier en grande difficulté GM\&S situé à La Souterraine dans la Creuse à aller trouver du travail à Ussel, situé à 140 kilomètres de là en Corrèze et à environ 2 h 30 de train ou de voiture. 
pendularité à grande vitesse (LANÉELLE, 2006), sur des situations de décohabitation conjugale pour raisons professionnelles (ORTAR, 2015), sur un cas de fermeture d'usine et de relocalisation des emplois (VIGNAL, 2005) ou encore de professions spécifiques comme les ouvriers de chantier (TESTENOIRE, 2006) ou les personnels aériens (LAMBERT, 2018), montrent notamment que la grande mobilité engage un travail quotidien d'ajustement au sein du couple et de la famille qui déborde largement sa seule dimension professionnelle. L'enjeu est donc de pouvoir étudier la grande mobilité domicile-travail en tant que révélateur des arbitrages professionnels, résidentiels et familiaux auxquels procèdent les individus.

Tout comme elle dépasse le seul cadre professionnel, la grande mobilité va au-delà de l'unique question des déplacements entre domicile et lieu de travail. Il s'agit de ne pas réduire l'étude des mobilités à celle des déplacements : le découplage entre domicile et lieu de travail peut en effet mener à une grande diversité de comportements de la part des individus, allant des navettes quotidiennes à la décohabitation conjugale hebdomadaire ou sur des périodes plus longues encore. Par définition, la grande mobilité correspond à des situations où les individus n'ont pas résolu la distance entre domicile et lieu de travail par une mobilité résidentielle et arbitrent en faveur de formes plus réversibles de mobilité (RAVALET et al., 2015) s'inscrivant dans une gamme d'arrangements divers allant de la pendularité à grande vitesse à la multilocalité résidentielle (BEAUVAIS et al., 2007) ou au télétravail (DUCHÊNE-LACROIX, 2013). Partant du constat que la grande mobilité domicile-travail se distingue d'autres formes de mobilité par l'impact qu'elle a sur les modes de vie des individus, on propose ici de considérer sur le plan analytique qu'une mobilité domicile-travail est « grande » si le découplage entre lieu de résidence et lieu de travail rend suffisamment coûteux (en temps, en argent, en fatigue, etc.) les déplacements entre domicile et lieu de travail pour qu'il soit trop difficile de les envisager sur une base quotidienne (LANDESMAN, SEWARD, 2013).

L'objectif de cet article est d'étudier la façon dont les arbitrages des individus en situation de grande mobilité s'inscrivent à l'articulation de logiques spatiales et socioprofessionnelles. Parce qu'elle peut être analysée à la fois comme ressource et contrainte, la mobilité doit être étudiée à l'aune des inégalités qu'elle produit ou qu'elle contribue à renforcer (ORFEUIL, RIPOLL, 2015 ; KORSU, WENGLENSKI, 2013). Pour ce faire, nous proposons une analyse systématique, à l'échelle de la France métropolitaine, des modalités de combinaison des inégalités sociodémographique et des logiques spatiales à l'œuvre dans la grande mobilité domicile-travail. Comment la grande mobilité domicile-travail organise-t-elle les inégalités entre travailleurs, et comment ces inégalités s'inscrivent-elles dans la grande mobilité ?

Le premier enjeu est d'ordre descriptif. En mobilisant les données du Recensement de la population (encadré 1), il est possible d'identifier, de caractériser, et de localiser les grands mobiles. Si elle fait l'objet de propositions de mesure (BRUTEL, 2011 ; Vincent-Geslin, KaUfMann, 2012 ; CoudÈne, Levy, 2016), la grande mobilité domicile-travail reste mal connue et souvent ramenée aux déplacements de cadres 
supérieurs circulant à grande vitesse entre métropoles. Le deuxième enjeu a une portée plus analytique. Les injonctions à la mobilité géographique adressées aux travailleurs reposent sur une conception appauvrie de la mobilité et des arbitrages qu'elle implique. La mobilité domicile-travail révèle un jeu complexe d'inégalités inscrites dans l'espace géographique comme dans l'espace social, inégalités qu'elle contribue aussi à recomposer.

Dans un premier temps, on présente la définition des grandes mobilités domiciletravail qui sera utilisée dans l'article et qui permet d'identifier 495000 individus en situation de grande mobilité en France métropolitaine. Dans un deuxième temps, la description des grandes mobilités selon différentes caractéristiques professionnelles,

\section{ENCADRÉ 1}

\section{Identifier les mobilités domicile-travail avec le Recensement de la population}

Le fichier détail « Mobilités professionnelles des individus : déplacements commune de résidence/commune de travail » est mis à disposition en libre accès sur le site internet de l'Institut national de la statistique et des études économiques (Insee). Il renseigne la commune de résidence et la commune de travail des actifs occupés. Il est issu du Recensement rénové de la population qui, depuis 2006, est annuel et couvre l'ensemble du territoire une fois tous les cinq ans. Le millésime 2015, utilisé dans cet article, est donc construit à partir d'enquêtes menées de 2013 à 2017.

Sont considérées comme domiciliées dans un logement donné les personnes vivant habituellement dans ce logement. Il s'agit principalement des habitants identifiés comme « permanents » du logement, à savoir les personnes qui vivent dans ce logement la plus grande partie de l'année. Il est précisé dans la feuille du questionnaire consacrée au logement que les « conjoints éloignés pour raisons professionnelles » sont considérés comme des habitants permanents. Réciproquement, les « personnes qui habitent ici pour des raisons professionnelles et retournent le week-end dans leur logement familial » ne remplissent pas de bulletin individuel pour ce logement.

Le lieu de travail est identifié par la question : «Quelle est l'adresse de votre lieu de travail ? » Pour les non-réponses et les individus indiquant un travail à domicile ou un lieu de travail variable, le lieu de travail est affecté à la commune de résidence.

L'adresse précise du lieu de résidence et du lieu de travail des individus n'étant pas diffusée, on ne peut pas mesurer précisément la distance entre domicile et lieu travail. Il est par ailleurs impossible d'évaluer l'accès des individus à différents moyens de transport et donc d'aborder la grande mobilité sous l'angle des temps de trajet comme le fait la littérature scientifique spécialisée (VINCENT-GESLIN, RAVALET, 2016).

Le Recensement ne permet pas non plus d'identifier les formes d'arrangement résidentiel que peuvent adopter les individus (pied-à-terre, nuitées occasionnelles, télétravail, etc.) et donc de faire la part de ce qui relèverait des déplacements et de la multilocalité résidentielle. Les données imposent donc de traiter la grande mobilité domicile-travail comme une situation de découplage entre lieu de résidence et lieu de travail, indépendamment des arrangements et des déplacements auxquels ont recours les individus. 
résidentielles et familiales montre que ces mobilités se superposent aux inégalités entre travailleurs et qu'elles mettent en jeu des arbitrages complexes qui dépassent largement la sphère professionnelle. On s'intéresse ensuite à la complexité des arbitrages sousjacents aux grandes mobilités : dans une troisième partie, on étudie l'inscription des mobilités dans des configurations spatiales entre centralité et périphérie ; puis, dans une quatrième partie, on explore l'espace des mobilités tel qu'il est défini par les types de circulation entre espaces. Cet espace est structuré par des inégalités socioprofessionnelles et de situation dans l'emploi qui invitent dans une dernière partie à une analyse toutes choses égales par ailleurs qui fait apparaître de nouvelles formes d'inégalités entre travailleurs, notamment de genre.

\section{Près de cinq cent mille actifs occupés en situation de grande mobilité domicile-travail en France métropolitaine}

On propose ici d'étudier la grande mobilité domicile-travail en mobilisant la catégorie statistique des « aires urbaines » (encadré 2). Les aires urbaines sont définies par la force d'attraction d'un pôle d'emploi dans lequel viennent travailler des actifs

\section{ENCADRÉ 2}

Le zonage en « aires urbaines », un outil d'analyse des relations entre centralité et périphérie

L'« aire urbaine » est une catégorie statistique élaborée par l'Insee en 1999 et rénovée en 2010. Il s'agit d'un ensemble de communes, d'un seul tenant et sans enclaves, constitué par un pôle urbain (composé d'un centre et de communes de banlieues) de plus de 10000 emplois et par des communes dites « périurbaines » dont $40 \%$ au moins de la population résidente travaille dans le pôle ou dans une commune satisfaisant elle-même ce critère.

Les communes appartenant à une aire urbaine mais ne faisant pas partie du pôle de cette aire urbaine en constituent la " couronne périurbaine ». Les communes dont au moins $40 \%$ des actifs occupés travaillent dans plusieurs grandes aires urbaines sans atteindre ce seuil avec une seule d'entre elles sont des communes périurbaines dites «multipolarisées ».

On considère ici que les communes faisant partie du pôle urbain d'une aire urbaine sont « centrales » et que les autres (communes périurbaines, communes rurales, villes isolées) sont « périphériques ». Toutes les communes situées hors d'une aire urbaine sont considérées comme «périphériques».

Par ailleurs, Chantal BRUTel (2011) distingue 12 aires «métropolitaines » (Paris, Lyon, Marseille, Lille, Toulouse, Nice, Bordeaux, Nantes, Strasbourg, Rennes, Grenoble et Montpellier) caractérisées par leur taille et par la concentration d'emplois de cadres des «fonctions métropolitaines » (conception-recherche, commerce inter-entreprises, culture-loisirs, etc.). Suivent 29 « grandes aires » qui comptent plus de 200000 habitants mais moins de 20000 cadres des fonctions métropolitaines. 
résidant dans des communes périphériques. Choisir la nomenclature des aires urbaines comme échelle d'observation des grandes mobilités domicile-travail permet d'observer en creux des grandes mobilités définies hors de la logique centripète sur laquelle cette nomenclature est construite. De plus, le zonage en aires urbaines permet de distinguer les espaces centraux et périphériques et donc de décrire les situations de mobilité domicile-travail en qualifiant les espaces dans lesquels elle s'inscrit. Enfin, utiliser le zonage en aires urbaines permet de naviguer entre trois niveaux d'analyse : un niveau agrégé à l'ensemble du territoire, la France métropolitaine ; un niveau localisé, à l'échelle de chaque aire urbaine ; et un niveau analytique en distinguant les espaces par leur caractère central ou périphérique.

La grande mobilité domicile-travail exclut les mobilités qui ont lieu au sein des aires urbaines entre le pôle et la périphérie (LE BRETON, 2008) ou entre polarités internes d'une même aire urbaine ${ }^{2}$ (Aguilera, 2005). Pour autant, le seul fait de travailler hors de son aire urbaine de résidence n'est pas tenu ici comme suffisant. En effet, les circulations d'individus entre aires urbaines limitrophes peuvent être longues et coûteuses tout comme elles peuvent correspondre à des « effets de frontière ». Un individu habitant dans une commune limitrophe d'une autre aire urbaine pourrait être considéré comme en situation de grande mobilité s'il va travailler dans cette autre aire urbaine. Se contenter de définir comme « grands mobiles» les individus travaillant hors de leur aire urbaine ou de leur espace de résidence aurait aussi pour effet de considérer comme en situation de grande mobilité tous ceux qui résident dans l'espace dit « multipolarisé », sous l'influence de plusieurs aires urbaines, et qui travaillent dans une aire urbaine proche.

L'objectif est donc ici d'identifier les mobilités domicile-travail qui puissent être qualifiées de « grandes », tout en proposant une définition analytique qui n'ait pas à mobiliser la grande diversité des configurations géographiques et urbaines des différents systèmes urbains dans lesquels résident les individus (morphologie, infrastructures de transport, conditions de circulation, etc.). On propose donc ici de suivre une démarche en deux temps :

- Pour les individus résidant dans une aire urbaine, la grande mobilité géographique est définie par le fait de travailler hors de son aire urbaine de résidence et dans un département non limitrophe de son département de résidence.

- Pour les individus résidant hors d'une aire urbaine, la grande mobilité géographique est définie par le fait de travailler dans un département non limitrophe de son département de résidence.

L'application de ce double critère permet d'éviter de compter comme grande mobilité des situations relevant de simples effets de frontière. Une aire urbaine donnée est en effet entourée d'un halo de communes limitrophes ou très proches dans lesquelles résident des actifs travaillant dans l'aire urbaine. Pour autant, pour une même

2. Une aire urbaine n'incluant pas toujours un unique pôle d'emploi. 
commune, leur nombre est insuffisant pour que ladite commune soit intégrée à l'aire urbaine. Les individus résidant dans l'une de ces communes et travaillant dans cette aire urbaine risquent d'être considérés comme en situation de grande mobilité du seul fait qu'ils ne travaillent pas dans le même espace que celui auquel appartient leur commune de résidence. La définition retenue ici permet d'éviter ces cas de faux positifs : par exemple, un individu résidant dans le département de l'Oise et dans l'aire urbaine de Beauvais ne sera pas considéré comme grand mobile s'il travaille dans les départements limitrophes du Val-d'Oise ou de la Seine-et-Marne.

Cette définition de la grande mobilité a cependant aussi pour effet de masquer des situations qui pourraient être considérées comme relevant de la grande mobilité. Ainsi, les individus résidant et travaillant dans l'aire urbaine de Paris ne sont pas considérés comme en situation de grande mobilité même si leurs lieux de travail et de résidence sont situés dans des départements non limitrophes. De même, tout en permettant de ne pas tenir pour grand mobile un individu résidant à Meaux (Seine-et-Marne) et travaillant dans l'Oise voisine, cette définition exclut aussi de la grande mobilité un individu travaillant dans l'Oise et résidant dans la ville de Nemours également située en Seine-et-Marne mais plus de 80 kilomètres au sud.

Par ailleurs, cette définition a pour conséquence que ne seront pas comptabilisés comme grands mobiles les individus résidant et travaillant dans des aires urbaines limitrophes comme celles de Lyon et de Grenoble ${ }^{3}$. Enfin, cette définition exclut les individus résidant et travaillant dans des réseaux d'aires urbaines situées dans des départements limitrophes, comme dans l'ouest de la France : on n'identifie ici que 1800 grands mobiles parmi les 11000 individus qui habitent à Rennes, Nantes, Le Mans, Angers, Tours ou Poitiers et qui travaillent dans une autre aire urbaine de cette liste.

La définition proposée ici n'a cependant pas que des effets restrictifs sur l'identification de la grande mobilité, notamment autour de l'aire urbaine de Paris qui s'étend sur plusieurs départements. Ainsi, les individus résidant dans un département limitrophe de l'aire urbaine de Paris pourront être considérés comme étant en situation de grande mobilité s'ils travaillent par exemple en Seine-Saint-Denis (18 000 individus en tout) ou dans le Val-de-Marne (6 000 individus). Cela dit, l'objectif n'est pas tant de couvrir tous les cas dans lesquels la mobilité domicile-travail peut être tenue pour pénible, coûteuse ou pesante pour les individus, comme le sont par exemple les grands déplacements internes à l'aire urbaine de Paris. Il s'agit plutôt de s'assurer que seules des mobilités ne pouvant pas être considérées autrement que comme longues soient prises en compte.

Dans l'ensemble, cette définition de la grande mobilité exclut des déplacements domicile-travail correspondant à des temps de trajet en voiture ou en train hors grande vitesse pouvant aller jusqu'à une heure et demie environ, comme c'est le cas entre le centre de Rennes et celui de Nantes ou, en Île-de-France, entre Rambouillet 
(Yvelines) et Melun (Seine-et-Marne $)^{4}$. Ce seuil empirique d'une heure et demie, soit trois heures aller-retour, ne recouvre qu' approximativement la définition proposée. On note cependant qu'il est plus restrictif que les seuils identifiés dans la littérature : si un trajet domicile-travail de 45 minutes, soit 90 minutes aller-retour, est souvent considéré comme la limite de ce que les individus peuvent envisager d'accepter, un trajet de 30 minutes a déjà un impact significatif sur les modes de vie (ÖHMAN, LiNDGREN, 2003 ; SANDOw, 2013). Sachant que l'analyse de la distribution des temps de déplacement journaliers établit qu'un seuil de 120 minutes par jour environ correspond à une « pendularité extrême » (JoLY, VincENT-GesLin, 2016), la définition qu'on propose de retenir ici est cohérente pour identifier les cas de « grande » mobilité domicile-travail.

Si les catégories statistiques et, plus encore peut-être, administratives ont leur part d'arbitraire, elles ont donc l'avantage de permettre d'élaborer avec une certaine économie de moyens une définition analytique et restrictive de la grande mobilité domicile-travail. Cette définition permet d'identifier 495000 actifs occupés en situation de grande mobilité, soit 1,9\% de l'ensemble des actifs occupés âgés de 15 ans ou plus résidant et travaillant en France métropolitaine (tableau 1).

L'intégration d'un département d'écart entre domicile et lieu de travail exclut de la définition près de 1,2 million d'individus travaillant dans un département limitrophe de celui de leur commune de résidence. Cependant, avec 1,9\% des actifs en emploi identifiés comme en situation de grande mobilité, soit près de 500000 individus, la définition retenue ici se situe entre le chiffre de 317000 individus travaillant à plus de 200 kilomètres de leur lieu de résidence (COUDÈnE, LeVy, 2016) et celui de $4 \%$

\section{TABLEAU 1 - Configurations domicile-travail des actifs occupés en France métropolitaine}

\begin{tabular}{lrr}
\hline Actifs occupés résidant dans une aire urbaine & & 21799000 \\
Travaillent dans leur aire urbaine de résidence & $86,3 \%$ & 18812000 \\
Travaillent dans une autre aire urbaine, dans le même département & $8,5 \%$ & 1851000 \\
Travaillent dans une autre aire urbaine, dans un département limitrophe & $3,4 \%$ & 733000 \\
Travaillent dans une autre aire urbaine, dans un département non limitrophe & $\mathbf{1 , 8 \%}$ & $\mathbf{4 0 3 0 0 0}$ \\
\hline Actifs occupés résidant hors d'une aire urbaine & & 3721000 \\
Travaillent dans leur département de résidence & $86,0 \%$ & 3200000 \\
Travaillent dans un département limitrophe & $11,5 \%$ & 429000 \\
Travaillent dans un département non limitrophe & $\mathbf{2 , 5 \%}$ & $\mathbf{9 2 0 0 0}$ \\
\hline Ensemble des actifs occupés & & 25520000 \\
Ensemble des individus en situation de grande mobilité domicile-travail & $\mathbf{1 , 9 \%}$ & $\mathbf{4 9 5 0 0 0}$ \\
\hline
\end{tabular}

Note : les lignes en gras correspondent aux situations de grande mobilité géographique domicile-travail.

Champ : individus actifs ayant un emploi et âgés de 15 ans ou plus, travaillant et résidant en France métropolitaine.

Source : Recensement de la population 2015, fichier détail « Mobilités professionnelles », Insee.

4. La définition retenue ici n'étant pas construite sur un critère kilométrique ou chronométrique, il ne s'agit pas là d'une règle absolue : les déplacements d'environ 900 individus résidant dans l'aire urbaine d'Orléans et travaillant dans celle de Tours ou dans le département de Paris, par exemple, sont comptés comme de grandes mobilités professionnelles quand bien même Orléans est à moins d'une heure et demie de Tours et de Paris par voie ferrée classique. 
d'individus connaissant un temps de trajet domicile-travail au moins égal à deux heures par jour en France (VInCENT-GesLin, KAUfMAnN, 2012) ${ }^{5}$.

\section{Segmentations socioprofessionnelles et arbitrages}

Au-delà de l'enjeu de mesure, il s'agit de voir comment la grande mobilité domiciletravail, telle qu'elle est définie ici, s'articule aux segmentations socioprofessionnelles.

En premier lieu, on constate que la grande mobilité se superpose aux inégalités socioprofessionnelles entre travailleurs (tableau 2). Ainsi, la part de grands mobiles est plus élevée chez les cadres, les hommes et les diplômés du supérieur alors qu'elle est faible parmi les employés, les femmes, les ouvriers, les individus les moins diplômés ou ceux employés à temps partiel ${ }^{6}$. Les cadres supérieurs disposent en effet de plus de ressources pour assumer les coûts financiers d'une grande mobilité (transports, hébergements temporaires, etc.) et les individus les plus qualifiés sont plus à même de tirer avantage, sur le plan salarial ou en matière de valorisation de compétences spécifiques, de la prise d'un poste très éloigné de leur domicile. Si la grande mobilité peut être un moyen d'accéder à des ressources d'ordre professionnel, d'autres mécanismes sont à l'œuvre. En effet, la part de grands mobiles est la plus élevée parmi les individus occupant un emploi à durée déterminée, alors qu'on pourrait s'attendre à ce

\section{TABLEAU 2 - Nombre et part de grands mobiles par variables socioprofessionnelles et sociodémographiques}

\begin{tabular}{lcc}
\hline Variables & Nombre de mobiles & Part de mobiles (en \%) \\
\hline Catégorie socioprofessionnelle & & \\
Agriculteurs exploitants & 2000 & 0,4 \\
Artisans, commerçants & 23000 & 1,4 \\
Cadres supérieurs & 162000 & 3,6 \\
Professions intermédiaires & 142000 & 2,1 \\
Employés & 86000 & 1,2 \\
Ouvriers & 80000 & 1,6 \\
\hline Secteur d'activité & & \\
Agriculture & 5000 & 0,7 \\
Industrie & 68000 & 2,1 \\
Construction & 34000 & 2,0 \\
Services & 278000 & 2,3 \\
Administration publique & 111000 & 1,4 \\
\hline
\end{tabular}

5. Ce qui équivaudrait, en généralisant rapidement à l'ensemble des actifs occupés, à un million de grands mobiles.

6. Les indépendants, et dans une plus grande mesure, les agriculteurs exploitants, se caractérisent par un faible taux de grande mobilité qui peut s'expliquer par le caractère fixe de leur patrimoine productif ainsi que par l'imbrication de leurs espaces résidentiels et professionnels. La catégorie socioprofessionnelle détaillée des individus n'étant pas diffusée, il n'est pas possible d'identifier plus précisément les 23000 grands mobiles de ce groupe. 


\begin{tabular}{|c|c|c|}
\hline Variables & Nombre de mobiles & Part de mobiles (en \%) \\
\hline \multicolumn{3}{|l|}{ Temps de travail } \\
\hline Temps plein & 434000 & 2,1 \\
\hline Temps partiel & 61000 & 1,4 \\
\hline \multicolumn{3}{|l|}{ Forme d'emploi } \\
\hline Emploi stable & 374000 & 2,0 \\
\hline Emploi précaire & 81000 & 2,4 \\
\hline Emploi indépendant & 40000 & 1,3 \\
\hline \multicolumn{3}{|l|}{ Sexe } \\
\hline Homme & 349000 & 2,7 \\
\hline Femme & 146000 & 1,2 \\
\hline \multicolumn{3}{|l|}{ Âge } \\
\hline$<30$ ans & 105000 & 2,1 \\
\hline 30-39 ans & 110000 & 1,8 \\
\hline $40-49$ ans & 133000 & 1,9 \\
\hline $50-59$ ans & 119000 & 1,9 \\
\hline 60 ans et plus & 29000 & 2,3 \\
\hline \multicolumn{3}{|l|}{ Type de ménage } \\
\hline Seul & 70000 & 1,7 \\
\hline Famille monoparentale & 37000 & 1,6 \\
\hline En couple sans enfant & 199000 & 2,0 \\
\hline En couple avec enfant(s) & 174000 & 2,1 \\
\hline Autre ménage & 15000 & 2,1 \\
\hline \multicolumn{3}{|l|}{ Diplôme } \\
\hline$<$ Baccalauréat & 148000 & 1,5 \\
\hline Baccalauréat & 96000 & 1,9 \\
\hline$>$ Baccalauréat & 251000 & 2,5 \\
\hline \multicolumn{3}{|c|}{ Statut d'occupation du logement } \\
\hline Propriétaire & 333000 & 2,2 \\
\hline Locataire & 107000 & 1,9 \\
\hline Locataire HLM & 38000 & 1,2 \\
\hline Autre & 18000 & 1,6 \\
\hline \multicolumn{3}{|l|}{ Type de logement } \\
\hline Maison & 342000 & 2,2 \\
\hline Appartement & 151000 & 1,6 \\
\hline Autre & 2000 & 1,6 \\
\hline
\end{tabular}

Note : le nombre d'enfants correspond au nombre d'enfants scolarisés vivant dans le logement.

Champ : individus actifs ayant un emploi et âgés de 15 ans ou plus, travaillant et résidant en France métropolitaine.

Source : Recensement de la population 2015, fichier détail « Mobilités professionnelles », Insee.

que l'emploi stable incite davantage les individus à accepter une situation de grande mobilité. Le logement et, d'une manière plus générale, l'ancrage territorial étant des ressources souvent mobilisées dans un contexte de fragilisation répétée de l'emploi salarié (VIGNAL, 2005), la grande mobilité serait ainsi moins un moyen d'accéder à un emploi stable qu'un moyen de ne pas subordonner les choix résidentiels à une trajectoire professionnelle précaire. 
C'est ce qu'illustre le cas des individus de moins de 30 ans. Débutant leur parcours résidentiel et familial, ils devraient être en mesure d'ajuster plus aisément leur lieu de résidence à leur lieu de travail que les actifs des autres catégories d'âge. Cependant, dans une situation en moyenne plus précaire vis-à-vis de l'emploi que les autres tranches d'âge, ils s'engagent dans une insertion professionnelle fragile sans vouloir lui subordonner leurs choix résidentiels, ce qui peut expliquer la part de grands mobiles parmi eux. Les comportements de mobilité sont en effet liés à la place des individus dans leur trajectoire résidentielle et familiale (SIGAUD, 2015). La multilocalité résidentielle est par exemple davantage le fait de travailleurs relativement âgés qui, arrivés à un stade tardif de leur parcours résidentiel, ne souhaitent plus déménager (AUTHIER et al., 2010) ou qui doivent composer avec l'ancrage territorial de leur conjoint et de leurs enfants. De fait, on compte 2,3\% de grands mobiles parmi les actifs occupés âgés de plus de 60 ans. La faiblesse des effectifs (8 500 individus environ) rend délicate l'analyse de leur profil mais les chefs d'entreprise et les cadres supérieurs y sont surreprésentés et plus de $80 \%$ des grands mobiles âgés de plus de 60 ans sont propriétaires de leur logement. Les emplois stables et le temps partiel sont aussi plus fréquents dans cette population, ce qui indiquerait que les plus âgés des grands mobiles sont en situation de transition de l'emploi vers l'inactivité.

La grande mobilité domicile-travail est donc associée à des situations professionnelles et personnelles contraintes, comme le fait d'occuper un emploi précaire plutôt que stable ou de vivre en couple avec enfant(s) plutôt que de vivre seul ou sans enfant. Ces arbitrages sont imbriqués dans les segmentations socioprofessionnelles : plus de $4,5 \%$ des cadres vivant en couple avec enfant(s) sont en situation de grande mobilité contre $1,8 \%$ des ouvriers. Pour les cadres supérieurs vivant en famille, donc, la grande mobilité domicile-travail est autant le produit de leur ancrage résidentiel que de leur capacité à circuler dans un marché du travail plus étendu géographiquement. La grande mobilité n'apparaît ainsi pas tant comme le produit de contraintes pesant sur les individus les moins bien dotés que comme une ressource que mobilisent les individus les mieux dotés pour chercher à concilier les dimensions professionnelles et privées de leur mode de vie.

\section{Des grandes mobilités inscrites dans des circulations entre centres et périphéries}

Traiter les données du Recensement en mobilisant le zonage en aire urbaine permet de qualifier les configurations spatiales différenciées dans lesquelles s'inscrivent les segmentations socioprofessionnelles que recouvre la grande mobilité. En premier lieu, 220000 individus en situation de grande mobilité, soit près de la moitié du total, travaillent dans l'aire urbaine de Paris (tableau 3). 
TABLEAU 3 - Localisation des individus en situation de grande mobilité géographique liée à l'emploi

\begin{tabular}{ccc|ccr}
\hline \multicolumn{5}{c|}{ Dix premières aires urbaines de résidence (origine) } & \multicolumn{3}{|c}{ Dix premières aires urbaines de travail (destination) } \\
\hline Aire urbaine & $\begin{array}{c}\text { Nombre } \\
\text { de mobiles }\end{array}$ & Part des mobiles & Aire urbaine & $\begin{array}{c}\text { Nombre } \\
\text { de mobiles }\end{array}$ & Part des mobiles \\
\hline Paris & 47500 & $9,6 \%$ & Paris & 220000 & $44,2 \%$ \\
Lyon & 18000 & $3,6 \%$ & Lyon & 23000 & $4,7 \%$ \\
Bordeaux & 10500 & $2,2 \%$ & Aix-Marseille & 12000 & $2,5 \%$ \\
Toulouse & 10200 & $2,1 \%$ & Toulouse & 9100 & $1,8 \%$ \\
Aix-Marseille & 10100 & $2,1 \%$ & Bordeaux & 7300 & $1,5 \%$ \\
Rouen & 9000 & $1,8 \%$ & Nantes & 6600 & $1,3 \%$ \\
Lille & 8700 & $1,8 \%$ & Lille & 5700 & $1,2 \%$ \\
Creil & 8100 & $1,6 \%$ & Rennes & 4400 & $0,9 \%$ \\
Tours & 7900 & $1,0 \%$ & Orléans & 4300 & $0,9 \%$ \\
Nantes & 7700 & $1,6 \%$ & Nice & 4200 & $0,9 \%$ \\
\hline
\end{tabular}

Champ : individus actifs ayant un emploi et âgés de 15 ans ou plus, travaillant et résidant en France métropolitaine. Source : Recensement de la population 2015, fichier détail « Mobilités professionnelles », Insee.

Le poids de l'aire urbaine de Paris dans les flux de grande mobilité s'explique par la concentration des emplois, notamment des emplois les plus qualifiés, et la forte tension sur les prix de l'immobilier résidentiel dans le pôle urbain de Paris. En ce qui concerne la grande mobilité liée à l'emploi, le bilan migratoire de l'aire urbaine de Paris est donc largement positif puisque 47500 des grands mobiles y résident alors que 220000 y travaillent, soit un solde migratoire apparent positif d'environ 172500 individus : si le lieu de travail de 44,2\% des grands mobiles est situé dans l'aire urbaine parisienne, ils ne sont que 9,6\% à y résider.

L'attractivité de l'aire urbaine de Paris recouvre trois logiques spatiales distinctes. Premièrement, 43500 des grands mobiles y travaillant résident en dehors d'une aire urbaine, principalement dans les départements de l'Oise, de l'Eure, de l'Aisne, de Bourgogne et d'Eure-et-Loir ceinturant l'Île-de-France. Deuxièmement, 33000 individus déclarent résider dans de grandes aires urbaines géographiquement éloignées et reliées par des lignes à grande vitesse : Lyon, Lille, Bordeaux, Tours, Aix-Marseille ou Nantes. Enfin, 27000 individus habitent dans des aires urbaines à proximité immédiate de l'Île-de-France : Creil, Rouen, Orléans, Chartres et Amiens. Les flux de grands mobiles en direction de Paris suivent donc trois logiques différentes : un phénomène d'extension par capillarité de l'attractivité de l'aire urbaine parisienne à l'espace hors aires urbaines aux marges du périurbain parisien, un phénomène d'attraction des pôles situés autour de l'aire urbaine de Paris, et des mobilités traversantes organisées autour des liaisons à grande vitesse qui relient Paris à de grandes aires urbaines métropolitaines.

Le fait que les grandes mobilités domicile-travail intègrent à la fois l'aire urbaine parisienne et les aires urbaines qui l'entourent est particulièrement visible dans le cas de Creil, qui compte 8100 actifs occupés travaillant dans un département non limitrophe de l'aire urbaine parisienne. À l'inverse, l'aire urbaine d'Orléans est la 
destination de près de 4500 grands mobiles dont 1100 résident dans l'aire urbaine de Paris et qui, par construction de la définition adoptée ici, ne peuvent pas résider dans les départements de l'Essonne ou de la Seine-et-Marne limitrophes du Loiret.

Si les flux restent incomparables à ceux qu'attire l'aire urbaine de Paris, on note en second lieu que 23000 grands mobiles travaillent dans l'aire urbaine de Lyon, soit près de $5 \%$ du total. Environ 4500 d'entre eux résident hors d'une aire urbaine, principalement dans les départements de la Drôme et de l'Ardèche ; 2800 habitent dans l'aire urbaine de Paris et 750 dans celle d'Aix-Marseille. Enfin, 2500 autres résident dans des aires urbaines relativement proches et bien reliées à celle de Lyon par le réseau routier et ferroviaire (Valence, Chambéry ou Aix-Marseille). D'une manière générale, les mobilités longues entre grandes aires urbaines géographiquement éloignées, hors Paris, sont rares : on compte 600 individus déclarant résider dans l'aire urbaine de Lyon et travailler dans celle de Toulouse, de Lille ou de Nice, et moins de 700 individus déclarant habiter dans l'aire urbaine de Toulouse et travailler dans celle de Lyon ou d'Aix-Marseille. Le cas de Lyon confirme donc les trois modalités d'organisation spatiale des grandes mobilités identifiée pour Paris : des mobilités traversantes entre grandes aires urbaines et Paris ou Lyon, des mobilités entre les grandes aires urbaines et les espaces situés à leurs marges, et des mobilités reliant les grandes aires urbaines à un réseau bien intégré d'aires urbaines de taille moyenne.

Au-delà des configurations territoriales propres à chaque aire urbaine, savoir précisément quelles sont, au sein de chaque aire urbaine, les communes de résidence et de travail des individus permet de détailler les types d'espaces entre lesquels se jouent les grandes mobilités de ces mêmes individus. Celles-ci ne sauraient être réduites aux circulations de centre-ville à centre-ville, qui se sont développées principalement le long des lignes à grande vitesse. Pour rendre compte de la diversité des configurations spatiales, les lieux de résidence et de travail des individus peuvent être séparés en deux catégories : les communes périphériques, qui regroupent le périurbain des aires urbaines ainsi que l'espace hors aires urbaines, et les communes des pôles qui rassemblent les communes du centre et de banlieue des aires. On peut ainsi distinguer quatre configurations spatiales de grande mobilité : les mobilités de pôle à pôle, de périphérie à pôle, de pôle à périphérie, et de périphérie à périphérie (tableau 4).

TABLEAU 4 - Circulations des grands mobiles par type d'espace de résidence et de travail

\begin{tabular}{l|ccc}
\hline \multirow{2}{*}{ Commune de résidence (origine) } & \multicolumn{3}{|c}{ Commune de travail (destination) } \\
\cline { 2 - 4 } & Pôles & Périphéries & Total \\
\hline \multirow{2}{*}{ Pôles } & 247000 & 45000 & 203000 \\
& $50 \%$ & $9 \%$ & $59 \%$ \\
\hline \multirow{2}{*}{ Périphéries } & 165000 & 38000 & 292000 \\
& $33,3 \%$ & $7,7 \%$ & $41 \%$ \\
\multirow{2}{*}{ Total } & 412000 & 83000 & 495000 \\
& $83,3 \%$ & $16,7 \%$ & $100 \%$ \\
\hline
\end{tabular}

Champ : individus actifs ayant un emploi et âgés de 15 ans ou plus, travaillant et résidant en France métropolitaine. Source : Recensement de la population 2015, fichier détail « Mobilités professionnelles », Insee. 
Si plus de $80 \%$ des grandes mobilités liées à l'emploi ont pour destination un pôle, les mobilités de pôle à pôle ne représentent que 50\% des grandes mobilités et $33 \%$ d'entre elles ont lieu entre une commune périphérique et le pôle d'une aire urbaine. Certes minoritaire, cette part est remarquable dans la mesure où la définition de la grande mobilité retenue ici n'inclut pas les mobilités qui correspondent au modèle centripète reliant les périphéries aux pôles. Parmi ces 33 \% de grandes mobilités périphérie-pôle, $18 \%$ ont pour origine le périurbain d'une aire urbaine et pour destination une commune n'appartenant ni au pôle de l'aire urbaine en question ni à celui d'une aire urbaine proche, mais dont l'influence est trop faible pour que la commune de résidence soit considérée comme faisant partie de son espace périurbain ou qualifiée de multipolarisée. Les $15 \%$ de mobilités périphérie-pôle restant correspondent à des mobilités en provenance de communes en dehors des aires urbaines et à destination d'un pôle d'une aire urbaine. Les mobilités de périphérie à pôle correspondent à des déplacements et à des arrangements résidentiels bien distincts de ceux qu'engagent les mobilités de pôle à pôle. Par ailleurs, si $83,3 \%$ des mobilités ont pour destination un pôle d'aire urbaine, il reste 83000 individus en situation de grande mobilité et travaillant dans un espace périphérique, sachant que les mobilités au sein d'espaces périphériques intégrés, par exemple entre les petits pôles urbains isolés et les communes rurales avoisinantes, ne sont pas incluses dans le champ. Les grandes mobilités de périphérie à périphérie constituent ainsi la modalité la plus éclatée, sur le plan spatial, à l'échelle du territoire métropolitain.

Les grandes mobilités consistent donc en un agencement diversifié de centralités et de périphéries. Prendre en compte la diversité de cet agencement invite à adopter un double niveau de lecture, spatial et social, et à étudier la façon dont les individus s'insèrent différemment dans ces configurations spatiales selon leurs caractéristiques socioprofessionnelles et sociodémographiques.

\section{Professionnelle, résidentielle et familiale : les trois logiques des grandes mobilités}

La mobilité domicile-travail est avant tout l'effet du découplage de la localisation des logements et de celle des emplois (BISAULT, 2017). Les pôles urbains concentrent les fonctions administratives et d'encadrement (FLOCH, LAINÉ, 2013), surtout dans les aires métropolitaines. À l'inverse, si l'emploi ouvrier n'a pas disparu des pôles urbains puisque plus de la moitié des ouvriers y travaillent, sa dynamique s'inscrit dans le tissu économique des espaces périphériques (Mischi et al., 2016). Un tel phénomène s'observe particulièrement dans les aires urbaines métropolitaines : moins de $15 \%$ des actifs occupés travaillant dans le pôle urbain de Paris et des autres aires urbaines métropolitaines sont des ouvriers. Enfin, les emplois occupés par des travailleurs des classes populaires et relevant de professions et catégories socioprofessionnelles distinctes ont des localisations différentes : $75 \%$ des employés travaillent dans un pôle urbain, là où sont regroupées la population et les entreprises consommatrices de services. 
Les lieux de résidence sont eux aussi très segmentés : alors que $79 \%$ des cadres résident dans le pôle d'une aire urbaine contre $53 \%$ des ouvriers, la moitié d'entre eux habitent dans le pôle d'une aire urbaine métropolitaine contre un cinquième seulement des ouvriers. Les professions intermédiaires et les employés sont nettement moins représentés que les cadres dans les centres des grandes aires urbaines et habitent plus souvent dans leurs banlieues que les ouvriers (Cusin et al., 2016). Même si la centralité urbaine peut être attractive pour les aménités qu'elle offre ${ }^{7}$, l'augmentation des prix de l'immobilier depuis le début des années 2000, à Paris notamment, durcit les conditions d'accès au logement. Elle contribue au filtrage social des résidents des espaces centraux ou de proche banlieue (DESPONDS, BERGEL, 2013) et contraint les membres des classes populaires qui souhaitent accéder à la propriété à s'éloigner des centres (ECKERT, URBAIN-MATHIS, 2019). Les espaces périphériques, et notamment le périurbain des grandes aires urbaines, ont été à ce titre investis par les classes moyennes et populaires (LAMBERT, 2015). Cependant, alors que les espaces centraux sont de plus en plus peuplés par des cadres supérieurs - on parle alors de phénomène de gentrification (CHABROL et al., 2016) -, les cadres peuvent aussi investir et s'investir dans les périphéries des aires urbaines (BACQUÉ et al., 2016).

Une analyse des correspondances fait apparaître un espace des grandes mobilités construit le long de deux axes (figure). L'axe 1, horizontal, oppose les mobilités de pôle à pôle, à gauche, et celles de périphérie à périphérie, à droite. L'axe 2, vertical, oppose les mobilités centripètes (périphérie-pôle), en bas, aux mobilités centrifuges (pôle-périphérie), en haut. L'espace dessiné par ces deux axes est organisé selon trois logiques : professionnelle, résidentielle et familiale. La logique professionnelle est exprimée sur l'axe 1 avec l'opposition entre cadres et ouvriers ainsi que sur l'axe 2 avec l'opposition entre emploi précaire et emploi stable ou à temps plein. La logique résidentielle est représentée sur l'axe 1 avec, d'un côté, les locataires du secteur privé ou d'un logement social et les individus vivant en appartement et, de l'autre côté, les propriétaires et les occupants d'une maison. Enfin, la logique familiale oppose sur l'axe 2 les individus habitant en couple avec ou sans enfant(s), ainsi que ceux âgés de 40 à 49 ans et de 50 à 59 ans, aux individus de moins de 30 ans et résidant seuls.

Ces trois logiques permettent de différencier quatre configurations spatiales de la grande mobilité domicile-travail. La logique professionnelle est déterminante pour distinguer les mobilités de pôle à pôle et les mobilités de périphérie à périphérie. Situées à gauche du repère, les mobilités pôle-pôle sont caractéristiques des cadres supérieurs, de diplômés de l'enseignement supérieur et d'actifs occupés dans le secteur des administrations publiques ${ }^{8}$. Il s'agit d'une population urbaine et surreprésentée dans les pôles ainsi qu'à Paris et dans les autres aires métropolitaines, occupant des

7. Bien que l'appétence pour la centralité urbaine soit caractéristique des classes supérieures du salariat (RÉRAT, 2016), les individus de classe populaire peuvent aussi faire preuve d'un attachement certain à des modes de vie centraux et urbains (DIETRICH-RAGON, 2014).

8. Si la part de ces individus n'est que de 1,4\% parmi les actifs occupés de l'administration publique, elle monte à $2,6 \%$ pour les cadres du secteur public. 
emplois eux aussi concentrés dans les pôles. Les mobilités de périphérie à périphérie, elles, sont caractéristiques des ouvriers, des individus peu diplômés et du secteur de l'industrie, des populations et des emplois surreprésentés dans les espaces périphériques. L'opposition entre les grandes mobilités des cadres (pôle-pôle) et des ouvriers (périphérie-périphérie) suit aussi une logique résidentielle, les grandes mobilités pôle-pôle correspondant à des modalités urbaines d'habitat comme le fait de vivre en appartement et d'être locataire (à plus forte raison locataire du parc social, particulièrement concentré dans les banlieues des grandes aires urbaines) et les grandes mobilités périphérie-périphérie correspondant à des formes de logement caractéristiques des espaces périphériques (maison, propriété).

L'opposition entre les grandes mobilités des cadres et des ouvriers ne passe pas particulièrement par les conditions d'emploi. Ces dernières contribuent à distinguer le long de l'axe 2 les mobilités de périphérie à pôle, situées en bas du repère, et les

\section{FIGURE - La configuration sociospatiale des grandes mobilités}

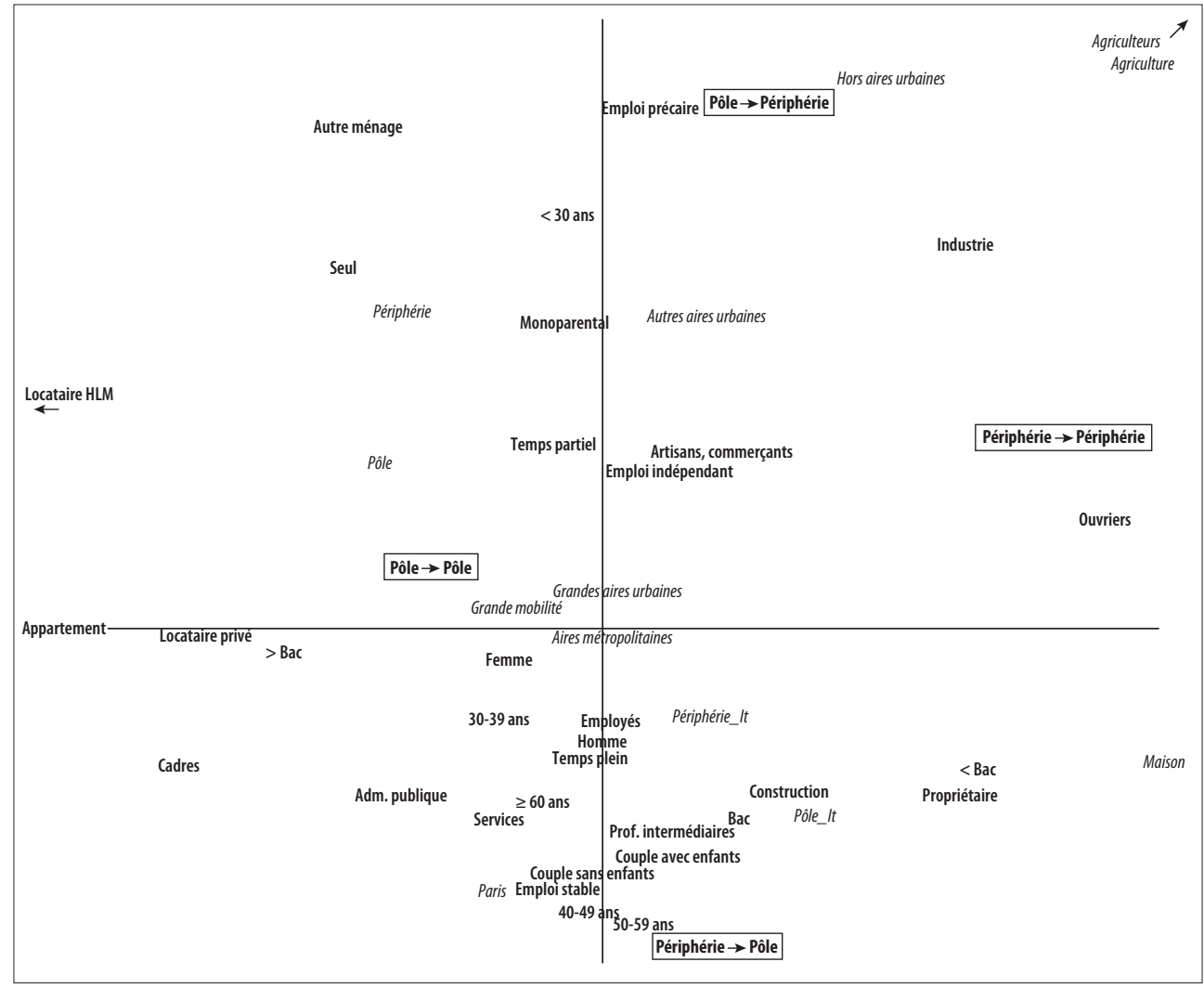

Note : les variables actives représentent la part de chaque type de mobilité (pôle-pôle, pôle-périphérie, périphérie-pôle, périphériepériphérie) parmi les grands mobiles par caractéristique représentée. Les modalités dont l'intitulé est en italique ont été traitées comme illustratives. Le suffixe «_lt » désigne la localisation de la commune où travaillent les individus.

Champ : individus actifs occupés en situation de grande mobilité, travaillant et résidant en France métropolitaine.

Source : Recensement de la population 2015, fichier détail « Mobilités professionnelles », Insee. 
mobilités de pôle à périphérie situées en haut. Les mobilités pôle-périphérie correspondent à l'emploi précaire et, dans une moindre mesure, à l'emploi à temps partiel. Les mobilités de pôle à périphérie se rapportent aussi aux individus jeunes, et à ceux qui vivent en famille monoparentale ou en ménage complexe. On peut donc y voir la forme de grande mobilité qui renvoie aux situations professionnelles et personnelles les plus fragiles, et qui a le plus de chances de représenter une contrainte venant s'ajouter à des modes de vie déjà contraints.

Ce sont finalement les grandes mobilités de périphérie à pôle (33\% des grandes mobilités) qui révèlent le mieux les arrangements entre vie professionnelle et vie privée. Sur le plan familial, elles concernent des individus d'âge intermédiaire (30-39 ans et 40-49 ans) vivant en couple avec ou sans enfant(s), autant de caractéristiques qui coïncident avec des localisations résidentielles dans la périphérie des aires urbaines leur permettant de desserrer les contraintes propres au marché du logement dans les pôles urbains (BONVALET, 2010). Sur le plan professionnel, les mobilités périphérie-pôle correspondent à des individus des classes moyennes dont la situation dans l'emploi est sécurisée : professions intermédiaires, niveau de diplôme moyen, emploi à durée indéterminée et emploi à temps plein. Se dessine ainsi le portrait de grands mobiles correctement dotés en ressources professionnelles mais devant composer avec le décalage entre le besoin d'accéder à des emplois urbains d'une part et la volonté d'accéder à des conditions de logement rendues possibles par la périphérie d'autre part. Les grandes mobilités périphérie-pôle dressent quant à elles un portrait très similaire à celui des « résidents "installés" » des zones pavillonnaires périphériques (GATEAU, 2017). Elles recouvrent donc des arbitrages à l'issue desquels l'implantation résidentielle serait en quelque sorte protégée par la grande mobilité domicile-travail. La vie en couple coïncide avec ce type de mobilité indépendamment du nombre d'enfants, ce qui donne à voir des individus dont les choix résidentiels sont contraints par la cohabitation conjugale, notamment en cas de couple biactif, et qui ne peuvent ou ne veulent ajuster leur lieu de résidence à leur lieu de travail. La configuration périphérie-pôle est la forme de mobilité la plus associée aux hommes et se rapporterait ainsi à un mode de vie familial organisé autour d'une répartition traditionnelle des rôles avec l'ancrage résidentiel et local des femmes d'une part et la grande mobilité domicile-travail des hommes d'autre part.

La déclinaison spatiale des grandes mobilités fait donc apparaître quatre types de grands mobiles bien différenciés : des mobiles urbains et protégés (cadres supérieurs, mobilité de pôle à pôle), des mobiles populaires périphériques (ouvriers, périphériepériphérie), des mobiles précaires sous contrainte (jeunes en emploi à durée limitée, pôle-périphérie) et enfin des mobiles moyens « installés » (professions intermédiaires, périphérie-pôle). La grande mobilité s'inscrit dans des logiques inégalitaires à la fois professionnelles, résidentielles et spatiales, dont on peut étudier les déterminants individuels. 


\section{Inégalités socioprofessionnelles et rapports de genre : deux clés de lecture pour expliquer la grande mobilité des salariés}

Un premier modèle de régression logistique estime la probabilité d'être en situation de grande mobilité plutôt que de ne pas l'être (tableau 5). Les variables explicatives retenues sont l'âge, la catégorie socioprofessionnelle, la situation dans l'emploi', le secteur d'activité, une variable croisant le sexe des individus et le type de ménage dans lequel ils s'inscrivent, le statut d'occupation du logement et enfin le type de logement. Ont été incluses comme variables de contrôle le type d'espace ainsi que le type d'aire urbaine dans lequel les individus résident (pôle urbain ou périphérie). Faisant double emploi avec la catégorie socioprofessionnelle, le diplôme n'a pas été retenu dans l'analyse. Enfin, les grandes mobilités des indépendants étant rares et délicates à interpréter en l'absence d'information détaillée sur leur activité, la population étudiée est ici restreinte aux salariés.

En contrôlant les caractéristiques socioprofessionnelles et sociodémographiques des individus, on note que la probabilité d'être en situation de grande mobilité plutôt que de ne pas l'être est plus élevée pour les individus résidant dans les aires urbaines non métropolitaines et dans l'espace hors aires urbaines que dans l'aire urbaine de Paris. On peut faire l'hypothèse que les mobilités vers Paris et sa banlieue, ou entre le périurbain parisien et les espaces qui l'entourent, ne sont pas comptabilisées comme des mobilités longues. La spécificité de l'Île-de-France, testée par deux estimations différentes, l'une portant sur les données de l'île-de-France uniquement, l'autre sur les données des autres régions, s'observe à deux niveaux. Premièrement, les ouvriers qui y habitent n'ont pas, toutes choses égales par ailleurs, une probabilité significativement différente de celle des professions intermédiaires d'être en situation de grande mobilité plutôt que de ne pas l'être. Deuxièmement, le fait d'habiter un appartement plutôt qu'une maison a, en Île-de-France, un effet légèrement positif sur la probabilité d'être en situation de grande mobilité plutôt que de ne pas l'être alors que cet effet est légèrement négatif pour les individus résidant dans une autre région. Puisque le modèle présenté dans le tableau 5 contrôle le type d'espace et l'aire urbaine de résidence, il n'identifie pas d'effet significatif du type de logement sur la probabilité d'être mobile. Enfin, on constate qu'être âgé de moins de 30 ans n'a qu'un effet très faible ; cela est cohérent avec le fait que ce qui est déterminant dans le cas des jeunes grands mobiles est leur situation professionnelle et familiale plus que leur âge. Par contre, être âgé de 60 ans ou plus a toujours un effet positif toutes choses égales par ailleurs, ce qui confirme l'analyse selon laquelle la façon dont les individus de cet âge se situent dans leur parcours résidentiel l'emporte sur leurs caractéristiques socioprofessionnelles.

Cette analyse permet de dégager deux résultats principaux relatifs à la façon dont la grande mobilité domicile-travail s'articule aux inégalités sociales : l'opposition

9. Variable synthétique prenant en compte les situations suivantes : emploi stable ou précaire, à temps plein ou à temps partiel. 
TABLEAU 5 - Estimation de la probabilité d'être en situation de grande mobilité plutôt que de ne pas l'être

\begin{tabular}{lc}
\hline Variables & Coefficient \\
\hline Âge (réf. = 40-49 ans) & $0,06^{* * * *}$ \\
$<30$ ans & $-0,12^{* * * *}$ \\
$30-39$ ans & $-0,05^{* * *}$ \\
$50-59$ ans & $0,18^{* * *}$ \\
60 ans et plus & \\
\hline Catégorie socioprofessionnelle (réf. = professions intermédiaires) & $0,79^{* * * *}$ \\
Cadres supérieurs & $-0,28^{* * *}$ \\
Employés & $-0,65^{* * *}$
\end{tabular}

Type d'emploi (réf. = stable à temps plein)

Précaire à temps partiel

Précaire à temps plein

$0,12^{* * * *}$

Stable à temps partiel

$0,37^{* * * * *}$

Secteur (réf. $=$ services)

Industrie

Construction

$-0,40^{* * * *}$

Administration publique

$-0,10^{* * * *}$

$0,17^{* * * *}$

$-0,33^{* * *}$

Type de ménage (réf. = homme seul)

Femme seule

Femme, famille monoparentale

$-0,38^{* * *}$

Homme, famille monoparentale

$-0,08^{* * *+1}$

$0,25^{* * * * * *}$

Femme, en couple sans enfant

$-0,36^{* * *}$

Homme, en couple sans enfant

Femme, en couple avec enfant(s)

$0,39^{* * * *}$

$-0,59^{* * * *}$

Homme, en couple avec enfant(s)

$0,38^{* * * * *}$

Autres ménages

$0,20^{* * * *}$

Statut d'occupation (réf. = propriétaire)

Locataire hors HLM

Autres statuts

Type de logement (réf. $=$ maison)

Appartement

Aire urbaine de résidence (réf. $=$ Paris)

Autres aires métropolitaines

Grandes aires urbaines

$0,40^{* * * *}$

Autres aires et hors aires urbaines

$0,57^{* * * *}$

Espace de résidence (réf. = centre d'une aire urbaine)

Banlieue d'une aire urbaine

Périurbain d'une aire urbaine

Hors aire urbaine

Constante

$-4,16^{* * * *}$

Note : estimation par régression logistique, $* * *$ : significatif au seuil de $1 \%, * *$ : significatif au seuil de $5 \%$, : significatif au seuil de $10 \%, \mathrm{n}=6612860$, pondération normalisée.

Lecture : un coefficient positif indique que les individus qui présentent cette caractéristique plutôt que la caractéristique de référence ont plus de chances, toutes choses égales par ailleurs, d'être en situation de grande mobilité plutôt que de ne pas l'être.

Champ : salariés âgés de 15 ans ou plus travaillant et résidant en France métropolitaine, hors type de logement « autres » (foyer, hôtel, meublé, etc.).

Source : Recensement de la population 2015, fichier détail « Mobilités professionnelles », Insee. 
entre cadres et ouvriers d'une part, et celle entre hommes et femmes d'autre part. L'importance de l'opposition entre cadres et ouvriers invite à poursuivre l' analyse par la mobilisation de variables qui ne sont pas incluses dans le modèle car non disponibles dans le Recensement. On pense ainsi en premier lieu au niveau de revenu, essentiel pour assumer les coûts financiers d'une grande mobilité ; également à la valorisation des mobilités professionnelles internes (à l'occasion d'une mutation) ou externes (à l'occasion d'un changement d'employeur), plus étendues sur le plan géographique, dont la rentabilité, en particulier sur le plan salarial, est incertaine pour les employés et les ouvriers (LEMISTRE, MAGRINI, 2008). Les cadres sont les premiers concernés par les injonctions à la mobilité, y compris géographique, au cœur des formes contemporaines de gestion des ressources humaines, et bénéficient plus facilement que les salariés d'exécution de certains modes d'organisation du travail, comme le télétravail, qui peuvent contribuer à leur faire considérer comme plus acceptables les situations de grande mobilité. (SCHMidT, RoBERT-DEMONTROND, 2012). Enfin, la grande mobilité domicile-travail peut mettre en cause les formes d'ancrage local au rôle central dans les modes de vie populaires et notamment ouvriers (FoL, 2009 ; VIGNAL, 2014) alors que les cadres supérieurs évoluent dans un réseau relationnel plus plastique et plus dispersé sur le territoire (BIDART et al., 2011).

Le deuxième résultat majeur concerne l'importance des inégalités de genre et celle des configurations familiales. Le taux de grande mobilité, on l'a vu, est nettement plus faible chez les femmes $(1,2 \%)$ que chez les hommes $(2,7 \%)$. S'il est plus élevé parmi les femmes cadres et diplômées ainsi que parmi celles qui résident hors des aires urbaines, la seule prise en compte de la variable « sexe » ne fait pas apparaître clairement les spécificités des grandes mobilités des femmes. On a donc construit une variable combinant le sexe et le type de ménage pour appréhender les configurations familiales comme échelle pertinente d'observation des inégalités de genre. En effet, les femmes doivent faire face à des « conflits de rôle » (que ce soit entre leurs rôles domestique et professionnel ou au sein de chacun de ces rôles) très différents de ceux auxquels sont confrontés les hommes (COLLET, DAUBER, 2010). Elles occupent plus souvent que les hommes des emplois « sédentaires » (TESTENOIRE, 2006) si bien que la «conciliation » entre vie professionnelle et vie familiale s'impose comme un impératif qu'elles peuvent tenir puisque la proximité entre leur lieu de résidence et leur lieu de travail est plus grande que celle des hommes. Même quand elles sont en situation de grande mobilité, les femmes maintiennent les arrangements traditionnels, restent investies dans l'organisation de la vie domestique et concentrent leur travail domestique sur leur temps de présence au foyer plus qu'elles ne le réduisent (LANÉELLE, 2006). Le cadre théorique classique, qui met en avant le fait que les rôles domestiques sont plus déterminants que les caractéristiques socioprofessionnelles pour expliquer la moindre mobilité domicile-travail des femmes (MADDEN, 1981), est confirmé par exemple par la plus grande sélection socioprofessionnelle des femmes en situation de grande mobilité domicile-travail par rapport aux hommes (SANDOW, 2013). 
Pour saisir cette segmentation genrée, il est nécessaire de ne pas étudier les hommes et les femmes uniquement en introduisant la variable « sexe », mais d'articuler le sexe aux configurations conjugales et familiales dans lesquelles sont insérés les individus. On a estimé l'effet de ces différentes configurations sur la probabilité pour les actifs occupés d'être en situation de grande mobilité, en prenant pour référence les hommes vivant seuls. Quel que soit le type de ménage, le fait d'être une femme diminue la probabilité d'être en situation de grande mobilité. Si les comportements de grande mobilité des hommes et des femmes peuvent paraître homogènes avant la mise en couple (KAUFMANN, AUDiKANA, 2017), les femmes ont toutes choses égales par ailleurs moins de chances d'être en situation de grande mobilité. L'effet est plus important pour les femmes vivant en couple avec des enfants scolarisés (coefficient de $-0,56)$ que pour les femmes vivant en couple sans enfant scolarisé $(-0,35)$, alors que les hommes vivant en couple avec ou sans enfant(s) scolarisé(s) voient leur probabilité d'être en situation de grande mobilité augmenter dans des proportions comparables (respectivement $+0,41$ et $+0,42$ ). La présence d'enfant(s) a donc un effet sur les comportements de mobilité des femmes mais pas sur ceux des hommes. Notre estimation met au jour l'effet différencié et inégalitaire des configurations domestiques et familiales sur la probabilité d'être mobile, d'autant plus remarquable ici que cet effet est contrôlé de la catégorie socioprofessionnelle. De plus, cet effet n'est, par construction, estimé que pour les actifs occupés, ce qui met de côté la question du moindre taux d'activité des femmes et celle des comportements de retrait du marché du travail en présence d'enfant(s). Si la grande mobilité peut être une ressource professionnelle pour les femmes, elle est négociée avec le conjoint et compensée par un rattrapage des tâches domestiques (LAMBERT, 2018), là ou celle des hommes se traduit plutôt par des formes de désengagement et de marginalisation vis-à-vis du foyer qui peuvent être contradictoires avec leurs aspirations mais qui ne leur sont pas moins spécifiques (ORTAR, 2015). Plus qu'un arbitrage entre trajectoire professionnelle et résidentielle, la moindre mobilité des femmes révèle aussi leur place dans une division sexuelle du travail qui contribue à les maintenir dans une position subordonnée à la fois dans la sphère professionnelle et dans la sphère domestique (BATTAGLIOLA, 1999). Entrer dans une situation de grande mobilité suppose une renégociation coûteuse des rapports au sein du couple et de la famille qui semble plus lourde quand la mobilité concerne les femmes : quand ils sont temporaires, les grands déplacements domiciletravail des femmes augmentent moins la probabilité de séparation des couples que ceux des hommes ; quand ils sont pérennes, ils la font augmenter davantage que ceux des hommes (SANDOW, 2013).

Afin de tester la façon dont ces inégalités se déclinent selon les quatre types de grande mobilité identifiés plus haut, un modèle de régression logistique généralisée portant sur la probabilité de connaître une grande mobilité périphérie-pôle, pôlepériphérie ou périphérie-périphérie plutôt que de pôle à pôle détaille les différentes configurations spatiales de grande mobilité (tableau 6). 
TABLEAU 6 - Estimation de la probabilité de connaître une forme de mobilité plutôt qu'une autre

\begin{tabular}{|c|c|c|c|}
\hline \multirow[t]{2}{*}{ Variables } & \multicolumn{3}{|c|}{ Type de grande mobilité (réf. = pôle-pôle) } \\
\hline & Périphérie-pôle & Pôle-périphérie & Périphérie-périphérie \\
\hline \multicolumn{4}{|c|}{ Âge (réf. = 40-49 ans) } \\
\hline$<30$ ans & n.s. & $0,09^{* * *}$ & $0,16^{* * *}$ \\
\hline $30-39$ ans & $0,07^{* * *}$ & $-0,04^{* *}$ & $0,06^{* *}$ \\
\hline $50-59$ ans & $-0,04^{* * *}$ & n.s. & $-0,07^{* * *}$ \\
\hline 60 ans et plus & $-0,06^{* *}$ & n.s. & $-0,17^{* * *}$ \\
\hline \multicolumn{4}{|c|}{ Catégorie socioprofessionnelle (réf. = professions intermédiaires) } \\
\hline Cadres supérieurs & $-0,34^{* * *}$ & $-0,54^{* * * *}$ & $-0,84^{* * *}$ \\
\hline Employés & $0,08^{* * *}$ & $0,18^{* * * *}$ & $0,10^{* * * *}$ \\
\hline Ouvriers & $0,24^{* * *}$ & $0,41^{* * * *}$ & $0,74^{* * *}$ \\
\hline
\end{tabular}

Type d'emploi (réf. = stable à temps plein)

\begin{tabular}{|c|c|c|c|}
\hline Précaire à temps partiel & $-0,05^{* *}$ & $0,09^{* * *}$ & n.s. \\
\hline Précaire à temps plein & n.s. & $0,24^{* * * *}$ & $0,23^{* * *}$ \\
\hline Stable à temps partiel & n.s. & $-0,1^{* * * *}$ & n.s. \\
\hline
\end{tabular}

\section{Secteur (réf. $=$ services)}

Industrie

Construction

$-0,1^{* * * *}$

n.s.

\begin{tabular}{|c|c|c|c|}
\hline Construction & n.s. & $-0,07^{* *}$ & $-0,11^{* * *}$ \\
\hline Administration publique & $-0,03^{* *}$ & $-0,35^{* * *}$ & $-0,33^{* * *}$ \\
\hline
\end{tabular}

Type de ménage (réf. = homme seul)

Femme seule

Femme, famille monoparentale

$\begin{array}{ccc}\text { n.s. } & \text { n.s. } & \text { n.s. } \\ -0,2^{* * * *} & 0,13^{* * *} & \text { n.s. } \\ 0,12^{* * * *} & \text { n.s. } & \text { n.s. }\end{array}$

Homme, famille monoparentale

Femme, en couple sans enfant

$0,13^{* * *}$

n.s.

$0,11^{* * * *}$

Homme, en couple sans enfant

Femme, en couple avec enfant(s)

$0,05^{* * *}$

$-0,13^{* * *}$

n.s.

$0,07^{* * * *}$

Homme, en couple avec enfant(s)

$-0,12^{* * *}$

$-0,17^{* *}$

$-0,22^{* * * *}$

Autres ménages

$-0,09^{* *}$

$-0,16^{* * *}$

$-0,16^{* * *}$

Statut d'occupation (réf. = propriétaire)

Locataire hors HLM $0,07^{*}$

Autres statuts $-0,26^{* *}$

n.s.

n.s.

Type de logement (réf. $=$ maison)

Appartement

$$
-1,01^{* * *}
$$

n.s.

$0,10^{* * *}$

Aire urbaine de résidence (réf. = Paris)

Autres aires métropolitaines

Grandes aires urbaines

Autres aires et hors aires urbaines

Constante

$-0,17^{* * * *}$
$-0,12^{* * *}$
$0,83^{* * *}$
$-1,42^{* * *}$

$-0,10^{* * *}$

$-1,02^{* * * *}$

Note : estimation par régression logistique généralisée, *** : significatif au seuil de $1 \%$, ** : significatif au seuil de $5 \%, *$ : significatif au seuil de $10 \%$, n.s. : non significatif ; $\mathrm{n}=130$ 541, pondération normalisée.

Lecture : un coefficient positif indique que les individus qui présentent cette caractéristique plutôt que la caractéristique de référence ont plus de chances, toutes choses égales par ailleurs, de connaître la forme de grande mobilité concernée plutôt qu'une grande mobilité de pôle à pôle.

Champ : salariés âgés de 15 ans ou plus travaillant et résidant en France métropolitaine et en situation de grande mobilité, hors type de logement « autres » (foyer, hôtel, meublé, etc.).

Source : Recensement de la population 2015, fichier détail « Mobilités professionnelles », Insee. 
L'estimation toutes choses égales par ailleurs de la probabilité de connaître une forme de grande mobilité domicile-travail plutôt qu'une autre confirme les segmentations mises en avant dans l'espace des grandes mobilités présenté dans la figure. Être employé plutôt que cadre a le même effet que le fait d'être profession intermédiaire sur chaque type de mobilité. L'âge contribue à distinguer les mobilités pôle-périphérie et périphérie-périphérie, plus spécifiques aux jeunes. Les mobilités de pôle à périphérie se caractérisent nettement par l'importance du statut d'emploi et, plus particulièrement, par le fait d'occuper un emploi précaire à temps plein. Chaque type de mobilité contribue à fixer les inégalités entre travailleurs, notamment autour de l'opposition entre ouvriers (périphérie-périphérie) et cadres (pôle-pôle). La grande mobilité peut être une ressource professionnelle pour les ouvriers quand elle leur permet de valoriser leurs compétences sur des marchés spécifiques impliquant une localisation fortement contrainte des sites de production, comme dans le cas des grands chantiers. Par leur grande mobilité professionnelle et les avantages qu'elle procure, les ouvriers sous-traitants du nucléaire accélèrent leur accès à la propriété et se mettent en couple plus rapidement. Ils se trouvent cependant de fait enfermés dans des mobilités entre espaces périphériques qui ne leur donnent d'autre choix que d'avoir recours à des formes d'arrangement qui finissent par peser sur leur investissement résidentiel et familial (GHIS MaLFILATRE, 2016).

La distinction des formes de grande mobilité met aussi en avant les différentes déclinaisons des inégalités de genre. La probabilité de connaître une mobilité pôlepériphérie, dont on a vu qu'elle correspondait à des situations plus précaires et à des choix plus contraints, ne dépend pas du sexe des individus et la présence d'enfants a le même effet pour les hommes que pour les femmes. La probabilité d'être en situation de mobilité périphérie-périphérie plutôt que pôle-pôle parmi les grands mobiles est ainsi moins influencée par le sexe que les autres formes de mobilité. Le fait d'être une femme en couple avec enfant(s) a certes un effet négatif sur la probabilité de connaître une mobilité périphérie-périphérie plutôt que pôle-pôle, mais c'est également le cas pour les hommes en couple avec enfant(s). Non seulement la conjugalité seule n'a pas d'effet différencié sur la probabilité des hommes et des femmes de connaître une forme de grande mobilité plutôt qu'une autre, mais les écarts entre les différentes formes de ménage sont dans l'ensemble relativement limités.

Ces résultats dessinent en creux la façon dont s'articulent les arbitrages professionnels et résidentiels, en particulier pour les grandes mobilités de pôle à pôle. En effet, être un homme ou une femme en couple sans enfant augmente la probabilité de connaître une autre forme de mobilité que celle de pôle à pôle. Par ailleurs, le fait d'être en couple avec enfant(s) diminue cette même probabilité. Par rapport aux autres grandes mobilités, les mobilités pôle-pôle sont associées à la présence d'enfant(s) dans les ménages. Ce résultat va à l'encontre de l'idée répandue selon laquelle la présence d'enfants concerne davantage des individus installés dans des espaces périphériques et allant travailler dans les pôles. La grande mobilité de pôle à pôle peut obéir aux mêmes logiques : quand des individus quittent la région parisienne pour s'installer dans une 
autre grande aire urbaine bien reliée à Paris, ils cherchent à rendre possibles leurs aspirations à un mode de vie urbain offrant des formes de centralité plus abordables qu'en région parisienne, notamment en matière de logement, tout en gardant un accès aux emplois concentrés autour de Paris. Tout en incitant les individus à choisir une grande mobilité de pôle à pôle, entre une grande aire urbaine et Paris, la présence d'enfant(s) a pour effet paradoxal d'alourdir les contraintes générées par cette grande mobilité.

L'analyse toutes choses égales par ailleurs fait donc plus que confirmer les inégalités socioprofessionnelles qui traversent les grandes mobilités domicile-travail. La grande mobilité est le produit d'un jeu complexe d'opportunités et de contraintes indissociables des configurations spatiales dans lesquelles elles sont ancrées et donc de la façon dont les individus inscrivent leurs modes de vie dans des territoires différenciés.

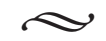

En mobilisant le Recensement de la population 2015, on peut identifier 450000 actifs occupés en situation de grande mobilité domicile-travail. Il s'agit d'individus qui, en France, travaillent dans une commune située à la fois dans une autre aire urbaine que celle dont relève leur commune d'habitation, et dans un département non limitrophe de celui dans lequel ils résident. Parce qu'elle est menée sur des données nationales et utilise une définition analytique de la grande mobilité, notre étude quantitative permet de combiner deux niveaux d'observation. Dans un premier temps, on constate que la grande mobilité recouvre les segmentations et les inégalités socioprofessionnelles qui parcourent les mondes du travail. Les grands mobiles sont en effet plutôt des hommes, cadres, qualifiés et insérés dans un réseau de mobilités organisées autour de Paris et des plus grandes aires urbaines. Dans un second temps, l'examen détaillé des caractéristiques socioprofessionnelles et sociodémographiques de ces grands mobiles montre l'importance des facteurs résidentiels et familiaux dans la distribution de la grande mobilité domicile-travail et, tout particulièrement, celle des choix que peuvent faire les individus entre mobilité professionnelle et immobilité résidentielle. Cet examen déconstruit la grande mobilité en révélant la diversité des logiques spatiales dans lesquelles elle s'inscrit.

Ces logiques spatiales sont elles-mêmes socialement différenciées. On a ainsi pu identifier quatre cas de figure très inégalement répartis dans l'espace social. Les mobilités de pôle à pôle, propres aux cadres supérieurs qualifiés, ne représentent que la moitié des grandes mobilités. Malgré une part de grands mobiles bien inférieure, les ouvriers participent à des mobilités entre espaces périphériques qui leur sont propres. Les couches moyennes du salariat, elles, cherchent à concilier un certain ancrage résidentiel dans les espaces périphériques et des mobilités longues tournées vers les pôles urbains. Si la grande mobilité domicile-travail a été définie ici de manière à la distinguer des mobilités centripètes propres à la croissance des aires urbaines et au phénomène de périurbanisation, elle n'en est pas moins soumise à des facteurs morphologiques d'articulation des centralités (où sont localisés les emplois qualifiés) 
aux périphéries (qui concentrent les logements abordables et les emplois ouvriers). L'approche typologique adoptée a d'ailleurs permis de faire émerger une forme à part de grande mobilité : les mobilités de pôle à périphérie, caractéristiques d'individus jeunes et à la situation dans l'emploi particulièrement fragile, et qui semblent être particulièrement contraintes.

C'est la prise en compte de la diversité des formes de circulation à l'œuvre qui permet de dépasser les lectures simplifiées de la grande mobilité, trop souvent ramenées aux déplacements à grande vitesse des cadres supérieurs circulant entre les espaces centraux des grandes aires urbaines. Si cette représentation correspond à un profil certes majoritaire de grands mobiles, la mobilité des ouvriers, des femmes et des jeunes peu qualifiés apparaît dans les interstices de la catégorie ici construite de grande mobilité et nous invite à nous interroger sur les arbitrages professionnels, résidentiels et familiaux qu'elle met en jeu. Il semble impossible de qualifier la grande mobilité des travailleurs et la façon dont elle s'inscrit dans des inégalités socioprofessionnelles et de genre tout en les recoupant, sans l'articuler à cette dimension spatiale spécifique.

En fin de compte, la grande mobilité domicile-travail recouvre des formes de circulation différenciées qui recoupent elles-mêmes des inégalités entre travailleurs profondément ancrées dans l'espace. Elle révèle l'inscription profondément différenciée des hommes et des femmes dans l'espace professionnel et dans l'espace résidentiel et domestique. Elle met également en lumière autant qu'elle catalyse les inégalités de genre qui la structurent en retour, et ne peut donc pas être analysée comme un arbitrage d'ordre uniquement professionnel. Si l'analyse toutes choses égales par ailleurs confirme la polarisation des comportements de mobilité entre cadres et ouvriers, elle expose surtout l'importance des configurations familiales et celle de leur déclinaison en matière de rapports de genre.

Un résultat central s'impose, qui contribue tant à une sociologie des mobilités qu'à une sociologie du travail et de l'emploi : la grande mobilité apparaît comme n'étant ni une contrainte écrasante, ni une opportunité libératrice. La façon dont les individus circulent entre les territoires est autant révélatrice des opportunités qu'ils cherchent à saisir que des contraintes avec lesquelles ils cherchent à composer. En cela, il est toujours aussi nécessaire de contester les discours qui font de la mobilité une disposition individuelle pour pouvoir désarmer les injonctions à la mobilité qui s'en nourrissent. Les perspectives de recherche ouvertes dans cet article sont liées à la nature des données mobilisées et au choix qui a été fait de proposer une définition analytique de la grande mobilité. Il conviendrait d'étudier les arrangements effectifs auxquels ont recours les individus en situation de grande mobilité domicile-travail, qu'ils soient d'ordre professionnel (télétravail), résidentiel (multilocalité résidentielle) ou relatifs à l'organisation des déplacements. Certains résultats, comme l'effet du logement social sur la grande mobilité ou encore la situation particulière des employés dans l'espace des mobilités, nécessitent de mobiliser des études empiriques plus détaillées pour être interprétés de manière satisfaisante. 


\section{BIBLIOGRAPHIE}

Aguilera A. (2005), "Growth in Commuting Distances in French Polycentric Metropolitan Areas: Paris, Lyon and Marseille”, Urban Studies, vol. 42, n 9, pp. 1537-1547.

Authier J.-Y (dir.), Bidet J., Collet A., Gilbert P., Steinmetz H. (2010), État des lieux sur les trajectoires résidentielles, La Défense, Plan urbanisme, construction architecture (PUCA).

Axisa J. J., Newbold K. B., Scott D. M (2012), "Migration, Urban Growth and Commuting Distance in Toronto's Commuter Shed”, AREA, vol. 44, n 3, pp. 344-355.

Bacqué M.-H., Charmes É., Launay L., Vermeersch S. (2016), «Des territoires entre ascension et déclin : trajectoires sociales dans la mosaïque périurbaine ", Revue française de sociologie, vol. $57, \mathrm{n}^{\circ} 4$, pp. 681-710.

Battagliola F. (1999), «Des femmes aux marges de l'activité, au cœur de la flexibilité », Travail, genre et sociétés, $\mathrm{n}^{\circ} 1$, pp. 157-177.

BeAuvais J.-M., Fouquet J.-P., AsSEGOND C. (2007), Recherche sur le développement de la grande vitesse et de la bi-résidentialité. Rentrer chez soi chaque soir ou une fois par semaine?, Paris, Ministère de l'Écologie, du Développement et de l'Aménagement durables.

Bidart C., Degenne A., Grossetti M. (2011), La Vie en réseau. Dynamique des relations sociales, Paris, Presses universitaires de France.

Bisault L. (2017), « Cadres et employés non qualifiés : les deux moteurs de l'emploi des territoires », Insee première, $\mathrm{n}^{\circ} 1674$.

Boltanski L., ChiAPEllo È. (1999), Le Nouvel Esprit du capitalisme, Paris, Gallimard.

Bonvalet C. (2010), « Les logiques des choix résidentiels des Franciliens », in Authier J.-Y., Bonvalet C., Lévy J.-P. (dir.), Élire domicile : la construction des choix résidentiels, Lyon, Presses universitaires de Lyon, pp. 55-76.

BRUTEL C. (2011), «Un maillage du territoire français. 12 aires métropolitaines, 29 grandes aires urbaines », Insee première, $\mathrm{n}^{\circ} 1333$.

Chabrol M., Collet A., Giroud M., Launay L., Rousseau M., Minassian H. T. (2016), Gentrifications, Paris, Éditions Amsterdam.

Collet B., Dauber A. S. (2010), “Gender and Job Mobility”, in Schneider N. F., Collet B., Mobile Living across Europe, vol. 2, Leverkusen Opladen, Barbara Budrich, pp. 173-194.

CONSEIL D'ORIENTATION POUR L'EMPLOI (2013), Emplois durablement vacants et difficultés de recrutement, rapport, Paris, Conseil d'orientation pour l'emploi.

Coudène M., Levy D. (2016), « De plus en plus de personnes travaillent en dehors de leur commune de résidence », Insee première, $\mathrm{n}^{\circ} 1605$.

Cusin F., Lefebvre H., Sigaud T. (2016), « La question périurbaine. Enquête sur la croissance et la diversité des espaces périphériques », Revue française de sociologie, vol. 57, $\mathrm{n}^{\circ} 4$, pp. 641-679.

DAVEZIES L. (2012), La Crise qui vient. La nouvelle fracture territoriale, Paris, Seuil.

DesPonds D., BERGEL P. (2013), « Transactions immobilières et substitutions socio-résidentielles en Seine-Saint-Denis », L'Espace géographique, vol. 42, n 2, pp. 115-127. 
Dietrich-RAGON P. (2014), « Résister à l'exil. Enquête auprès des mal-logés parisiens », Espaces et Sociétés, n ${ }^{\circ} 156-157$, pp. 19-35.

DuCHÊNE-LACROIX C. (2013), «Éléments pour une typologie des pratiques plurirésidentielles et d'un habiter multilocal », e-Migrinter, $\mathrm{n}^{\circ}$ 11, pp. 151-167.

ECKert H., Urbain-Mathis M. (2019), «Quand les ménages populaires s'installent dans leur maison : mobilités résidentielles et pratiques de sociabilité », Sociologie, vol. $10, \mathrm{n}^{\circ} 1$, pp. 52-72.

Floch J.-M., LAINÉ F. (2013), « Les métiers et leurs territoires », Insee première, n ${ }^{\circ} 478$.

Fol S. (2009), La Mobilité des pauvres. Pratiques d'habitants et politiques publiques, Paris, Belin.

Fol S., Miot Y., Vignal C. (dir.), (2014), Mobilités résidentielles, territoires et politiques publiques, Villeneuve-d'Ascq, Presses universitaires du Septentrion.

GATEAu M. (2017), « Les trajectoires résidentielles des pavillonnaires », SociologieS [en ligne]. http://journals.openedition.org/sociologies/5949

Ghis Malfilatre M. (2016), «L’impossible confinement du travail nucléaire. Expérience professionnelle et familiale des salariés sous-traitants exposés à la radioactivité », Travail et Emploi, n ${ }^{\circ} 147$, pp. 101-124.

Gomel B., MéDA D (2014), «Le RSA : un dispositif inadapté », La Vie des idées [en ligne], 11 mars. https://laviedesidees.fr/Le-RSA-un-dispositif-inadapte.html

Joly I, VinCENT-GeSLIN S (2016), “Intensive Travel Time: An Obligation or a Choice?”, European Transport Research Review, vol. 8, $\mathrm{n}^{\circ}$ 10, pp. 2-14.

Kaufmann V. (2017), Les Paradoxes de la mobilité : bouger, s'enraciner, $3^{\mathrm{e}}$ éd., Lausanne, Presses polytechniques et universitaires romandes.

Kaufmann V., Audikana A. (2017), Mobilité et libre circulation en Europe : un regard suisse, Paris, Economica, Lausanne, Fondation Jean Monnet pour l'Europe.

KLIS M. VAN DER, KARSTEn L. (2009), “The Commuter Family as a Geographical Adaptive Strategy for the Work-Family Balance”, Community, Work \& Family, vol. 3, n ${ }^{\circ}$ 12, pp. 339-354.

Korsu E., Wenglenski S. (2013), « Distance physique, proximité sociale et inégalités devant le chômage », in Fol S., Lehman-Frisch S., Morange M. (dir.), Ségrégation et justice spatiale, Nanterre, Presses universitaires de Paris Ouest, pp. 271-290.

LAMBerT A. (2015), «Tous propriétaires ! ». L'envers du décor pavillonnaire, Paris, Seuil.

LAMBert A. (2018), « Les incidences biographiques du travail mobile. Configurations familiales et rapports de genre », L'Année sociologique, vol. 68, n 2, p. 315-340.

Landesman J., Seward R. R. (2013), "Long Distance Commuting and Couple Satisfaction in Israel and United States: An Exploratory Study", Journal of Comparative Family Studies, vol. $44, n^{\circ} 6$, pp. 765-781.

LANÉELLE X. (2006), « Navette domicile-travail à grande vitesse : situation d'exception, arrangement traditionnel », Cahiers du genre, n 41, pp. 159-180. 
Le Breton É. (2008), Domicile-travail : les salariés à bout de souffle, Paris, Les Carnets de l'info.

LEMistre P. Magrini M.-B. (2008), « La mobilité géographique des jeunes ouvriers et employés est-elle rentable ? », Économie et Prévision, $\mathrm{n}^{\circ} 185$, pp. 63-88.

Madden J. F. (1981), "Why Women Work Closer to Home”, Urban Studies, vol. 18, n², pp. 181-194.

Mischi J., RenAhy N., Diallo A. (2016), « Les classes populaires en milieu rural », in Blancard S., Détang-Dessendre C., Renahy N. (dir.), Campagnes contemporaines. Enjeux économiques et sociaux des espaces ruraux français, Versailles, Éditions Quæ, pp. 23-34.

Moscovici S. (1959), «La résistance à la mobilité géographique dans les expériences de reconversion », Sociologie du travail, vol. 1, n 1, pp. 24-36.

Öhman M., Lindgren U. (2003), "Who Are the Long-Distance Commuters? Patterns and Driving Forces in Sweden", Cybergeo. European Journal of Geography [en ligne], document 243. https://doi.org/10.4000/cybergeo.4118

ORFEUIL J.-P., RipOLl F. (2015), Accès et mobilités : les nouvelles inégalités, Gollion, Infolio.

ORTAR N. (2015), La Vie en deux. Familles françaises et britanniques face à la mobilité géographique professionnelle, Paris, Pétra.

Ravalet E., Vincent-Geslin S., Kaufmann V., Viry G., Dubois Y. (2015), Grandes mobilités liées au travail : perspective européenne, Paris, Economica.

RÉRAT P. (2016), « Motivations résidentielles et pratiques de mobilité des classes moyennes supérieures dans les viles suisses », Espaces et sociétés, n 164-165, pp. 159-178.

SANDOw E. (2013), "Til Work Do Us Part: The Social Fallacy of Long-distance Commuting”, Urban Studies, vol. 51, $\mathrm{n}^{\circ}$ 3, pp. 526-543.

Schmidt C., Robert-Demontrond P. (2012), « Territoire et mobilité géographique infranationale. Étude des représentations et des trajectoires des salariés », Revue française de gestion, $\mathrm{n}^{\mathrm{o}} 226$, pp. 107-120.

Sigaud T. (2015), «Accompagner les mobilités résidentielles des salariés : l'épreuve de 1'“entrée en territoire” ", Espaces et sociétés, n 162, pp 129-145.

Testenoire A. (2006), «Éloignés au quotidien et ensemble. Arrangements conjugaux en milieu populaire », Cahiers du genre, $\mathrm{n}^{\mathrm{o}} 1$, pp. 117-138.

Vignal C. (2005), «Logiques professionnelles et logiques familiales : une articulation contrainte par la délocalisation de l'emploi », Sociologie du travail, vol. 47, n 2, pp. 153-169.

Vignal C. (2014), «L'ancrage local, une ressource pour les classes populaires des territoires désindustrialisés ? », in Fol S., Miot Y., Vignal C. (dir.), Mobilités résidentielles, territoires et politiques publiques, Villeneuve-d'Ascq, Presses universitaires du Septentrion, pp. 197-210.

Vincent-Geslin S., Kaufmann V. (2012), Mobilité sans racines, Paris, Descartes \& Cie.

Vincent-Geslin S., RAVALET E. (2016), "Determinants of Extreme Commuting: Evidence from Brussels, Geneva and Lyon”, Journal of Transport Geography, vol. 54, Issue C., pp. 240-247. 